\title{
Brain Computer Interface: EEG Signal Preprocessing Issues and Solutions
}

\author{
Nelly Elsayed \\ Center for Advanced Computer \\ Studies (CACS) \\ University of Louisiana at \\ Lafayette, Lafayette, LA, USA
}

\author{
Zaghloul Saad Zaghloul \\ Center for Advanced Computer \\ Studies (CACS) \\ University of Louisiana at \\ Lafayette, Lafayette, LA, USA
}

\author{
Magdy Bayoumi \\ Center for Advanced Computer \\ Studies (CACS) \\ University of Louisiana at \\ Lafayette, Lafayette, LA, USA
}

\begin{abstract}
Brain Computer Interface (BCI) is often directed at mapping, assisting, or repairing human cognitive or sensory-motor functions. Electroencephalogram (EEG) is a non-invasive method of acquisition brain electrical activities. Noises are impure the EEG recorded signal due to the physiologic and extra-physiologic artifacts. There are several techniques are intended to manipulate the EEG recorded signal during the BCI preprocessing stage of to achieve preferable results at the learning stage. This paper aims to present an overview on BCI different EEG brain signal recording artifacts and the methodologies to remove these artifacts from the signal focusing on different novel trends at $\mathrm{BCI}$ research areas.
\end{abstract}

\section{General Terms}

Brain Computer interface, EEG Signal Filtering, Machine learning.

\section{Keywords}

Brain Computer interface (BCI), EEG, artifact removal, preprocessing, EMG, EOG, filtering.

\section{INTRODUCTION}

The BCI research has arisen at the last decade. BCI research and development has focused primarily on neuro-prosthetics applications that aim to restore damaged hearing, sight and movement [1]. Nowadays, BCI research reached remarkable results at robot control $[6,8,9,12]$, motor disabilities recovery using BCI chip implants $[1,4,5,7,11]$, medical diagnoses and prediction $[15,18]$, security and authentications [14] and game controlling [10, 16, 17]. Figure 1 illustrates different BCI research.

\begin{abstract}
Brain cells communicate with each other through electrical impulses that structure the brain signal. There are several techniques for brain signal acquisition: invasive, semiinvasive, and non-invasive [10, 19]. EEG technique (which this paper concern) is a non-invasive technique for brain signal acquisition. It records brain signal along the scalp through measuring voltage fluctuations accompanying neurotransmission activity within the brain. The main advantages of EEG are that it is non-invasive so that there is no surgical intervention needed to set the electrodes on the brain, relative ease of use, portable, excellent time resolution (it gives the ability to see brain activity as it unfolds in real time, at the level of milliseconds (thousandths of a second)), low-cost comparing to the other brain signal acquisition techniques. The disadvantages of EEG that its very small signal to noise ratio and spatial localization resolution represent a limitation compared to other brain signal acquisition methods. According to the very small signal to noise at the EEG signal recorded, it cannot be directly used at processing stage such as feature extraction and pattern recognition directly at the BCI system. The EEG recorded signal has to pass through filtering and artifact removing at the preprocessing stage at BCI system. Figure 2 illustrates the BCI system stages and its components [20].
\end{abstract}

This paper organized as follows: Section 2 explains different kinds of artifacts that contaminated the brain signal acquired using EEG. Section 3 shows the different methodologies at novel BCI applications that targeting these artifacts removal, finally a brief conclusion at section 4 .

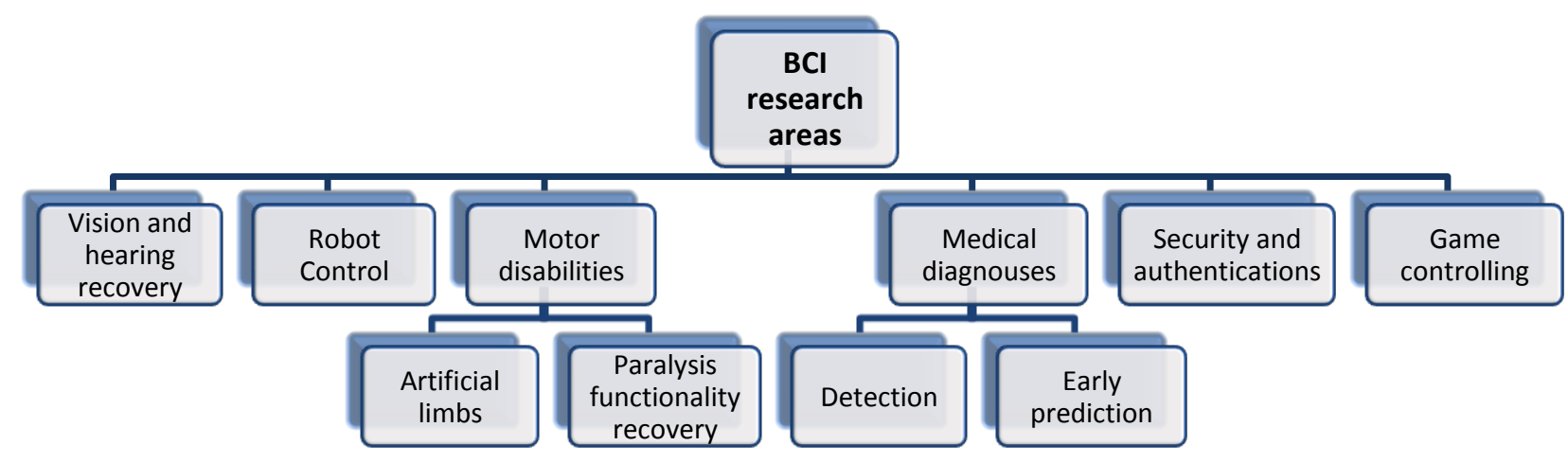

Fig 1: BCI research areas 


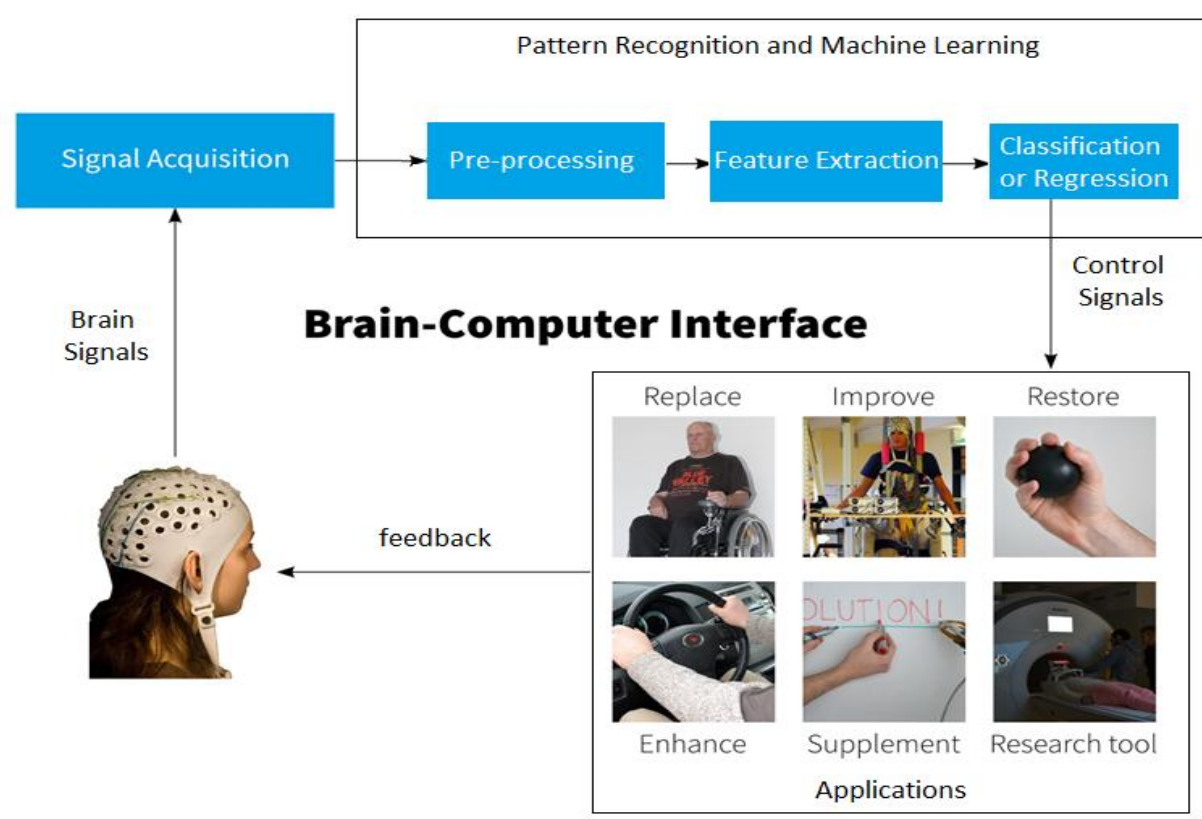

Fig 2: BCI Stages

\section{EEG ARTIFACTS}

EEG recorded signal artifacts are all the signals that contaminated the brain signal during the process of its acquisition. There are two types of artifacts: extraphysiologic and physiologic artifacts. Extra-physiologic artifacts are occurred due to external environment rather than the human body during brain signal acquisition such as (environment, equipment). Physiologic artifacts occurred due to the human body, and arose from sources other than the brain such as (eye blink, eyeball movement, breath, heart beats, muscles movement) [20].

\subsection{Extra-Physiologic Artifacts}

\subsection{1 (60-Hz) Alternating Current Artifact}

The $60-\mathrm{Hz}$ alternating is one EEG acquisition technical preparation problems. It happened when there is no sufficient grounding of the EEG cap electrodes on patient's head. The impedance between the ground of the amplifier and the active electrodes of the EEG cap becomes considerably large. At this case, the ground acts like an electrode which produces $60-\mathrm{Hz}$ artifact and causes indistinct signal recording.

\subsubsection{Electrode Artifacts}

Electrode popping and incorrect location of EEG electrode placement are the most common electrode artifacts at EEG brain signal acquisition. Incorrect location of EEG electrode placement is considered as technical preparation problem. Incorrect placement of the electrode on patient's head generates an incorrect acquisition of EEG signal. Electrode popping artifact happened due to abrupt impedance change at the acquiesced signal. It could be identified visually as it causes multiple sharp waveforms that bind to the EEG signal [25].

\subsubsection{Movements in recording environment}

Any movement of other persons around the patient can generate artifacts at signal recording, usually this artifact of capacitive or electrostatic origin. This should be avoided as much as possible, if avoidance is not possible as in the operating room and the intensive care unit (ICU), proper notation (remarks) must be placed on the records of the signal recorded.

\subsubsection{Interference of other Equipment}

This kind of artifacts is also named as powerline noise. Highfrequency radiation from different devices such as radio, television, cell phone, hospital paging systems, light florescent, and equipment which is captures by the electrode interference can overload EEG amplifiers. In this case, the EEG signal cannot be recorded because pens may fully be deflected by swerve upward or downward. The optimal solution to solve this problem is turn off these different devices during EEG machine recording the brain signal.

\subsection{Physiologic Artifacts}

\subsubsection{Electrooculography (EOG)}

EOG is the signal artifact occurs from eye movement functionality during EEG recording. EOG overlapping the EEG brain recorded signal. It is useful only in the medical case of identifying sleep stages at the EEG signal. Bell phenomena is observed by the vertical eye movements (i.e. eye blinks) which producing symmetric downward deflections for the front polar electrodes recording while the lateral eye movements affect lateral frontal electrodes recording [21].

\subsubsection{Electrocardiogram ( $E K G$ or $E C G$ )}

ECG (or EKG) artifacts are considered as the largest artifacts that could be found in the EEG recorded signal especially for those patients having wide and short necks. ECG is caused by the interference of the heart beats and moves potentials over patient's scalp surface [24]. Due to the regularity (rhythmicity) and coincidence of the ECG artifact, it could be easily detected and recognized through the EEG signal recording. The problem of detecting this artifact occurs at the case when the cerebral abnormal activity overlapped with other EEG signal artifacts and its appearance could be overlooked [25].

\subsubsection{Pulse Artifact}

There is a direct relationship between the pulse waves and the ECG. Pulse waves artifact occurs when the EEG electrode in places over a pulsating vessel causes slow waves that may simulate EEG activity [25]. The easiest solution to reduce this artifact is by replacing the electrode location on the scalp. 


\subsubsection{Skin artifacts}

Skin sweat (perspiration or diaphoresis) and not clean hairy leather of the patient are the major skin artifacts. Sweat is a fluid that secreted by sweat glands. Sweat major components are lactic acid and sodium chloride that causing enormous slow baseline due to their reaction with electrodes metals [24]. The hairy leather artifact increases the impedance between the electrode and the skin which effect on the recorded EEG signal correctness.

\subsubsection{Glossokinetic artifact}

This artifact is generated by the movement of the tongue. Glossokinetic artifact is similar to the EOG artifact caused by the eye movement although it is less steep than that produced by eye movement artifacts [26]. Glossokinetic artifact has a broad potential field that drops from frontal to occipital areas. In this artifact the amplitude of the parasagittal is smaller inferiorly than in potentials regions.

\subsubsection{Electromyogram (EMG)}

EMG is the signal generated by muscles movement. The most common artifact effect on EEG signal is the myogenic potentials that are caused by clenching of jaw muscles (frontalis and temporalis muscles). The potentials generated by the brain are longer duration than those that are generated by muscles. These artifacts are identified on the frequency, duration, and morphology of the signal [13]. Frontalis and temporalis muscles (e.g., clenching of jaw muscles) are common causes. Generally, the potentials generated in the muscles are of shorter duration than those generated in the brain and are identified easily on the basis of duration, morphology, and rate of firing (i.e., frequency) [26].

\section{ARTIFACTS REMOVAL}

At the BCI system, EEG signals filtering done at the preprocessing stage. There are several approaches that are designed to eliminate the noise that generated by various artifacts from the EEG signal. This section demonstrates different type of most remarkable filters approaches that are used in at BCI applications.

\subsection{Artifacts Avoidance}

The idea here is to remove the extra-physiologic artifacts by correctly assigning the EEG cap on the patient and preparing the place of the recording of other devices that can effect on the EEG signal acquisition from the patient. It is not an algorithm to be applied but it is a technical preparation for acquisition a high quality record before signal filtering from physiologic artifacts.

\subsection{Linear Filtering}

Linear filtering is commonly used for removing artifacts located in certain signals that do not have any overlapped frequency bands with the frequency of the brain signals. Lowpass filtering and high-pass filtering are the most noticeable examples for linear filtering algorithms [23]. High-pass filter is commonly used to remove the EOG artifacts as the EOG artifacts consist of low frequency components. Low-pass filter may remove most of EMG artifacts as most of EMG components have high-frequency values.

Ease implementation from software and hardware perspectives is one of the major advantages of linear filter. In addition, it does not need any extra information about the artifact itself, for example, to remove the EOG or the EMG noises from the EEG signal the linear filter does not need predefining of their signal information [2]. The major drawback of linear filter that in case of any overlapping between either both EEG and EOG (or EMG) signals or both EMG and EOG signals, the filter fails to filter the EEG signal and could not remove the EOG and EMG artifacts from the EEG signal.

\subsection{Linear Adaptive Filter}

Linear-adaptive filter is introduced at [2] is mainly based on the optimization theory and how the filter design can modify its propertied according to the selected feature from the signal being analyzed. The basic idea of the adaptive filter is to adapt the frequency response (coefficient) of the linear filter to generate a signal similar to the noise existing in the signal to be filtered. This linear-adaptive filter algorithm as considers that there are two signals primary signal and secondary signal, $d(n)$ and $x(n)$ respectively. $H(z)$ is a linear filter which produces an output $\mathrm{y}(\mathrm{n})$. To compute the error $\mathrm{e}(\mathrm{n})$ the output value $\mathrm{y}(\mathrm{n})$ is subtracted from the primary signal $d(n)[2,23]$. Figure 3 illustrates the linear-adaptive basic structure. The two major processes of this filtering algorithm are: filtering process and adaptive process. At the filtering process the output signal is the response of a digital filter. In this process, FIR filters are commonly used because they are simple, linear and stable filters. At the adaptive process, The adjustment of transfer function $\mathrm{H}(\mathrm{z})$ is according to an optimizing algorithm. This adaptation is directed by the primary signal and the filter output difference (error) value.

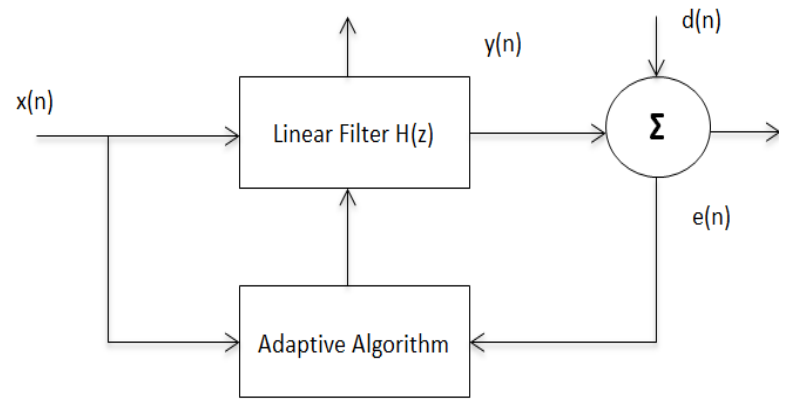

Fig 3: The linear-adaptive filter structure

3.4 Quantum Neural Network-Based Filter

Due to Moore's law, the interest of finding new hardware design rather than the classical computers led to various innovations at quantum computations and quantum inspired algorithms. Quantum inspired algorithms achieved remarkable results at various algorithms [3]. One example of quantum inspired algorithms for EEG signal filtering is the recurrent quantum neural network (RQNN). RQNN filter improves the acquired EEG brain signal quality and segregation of signal artifacts. RQNN filter mechanism is combining between both the recurrent neural networks (RNN) and quantum computer architecture mechanisms and concepts. RQNN quantum mechanism which based on Schrodinger wave equation trigged the recurrent neural network to efficient dealing with the EEG signal filtering, in addition that the RNN the is using an unsupervised learning algorithm, it enables the filter to be a powerful tool for capturing the EEG brain signal statistical behaviors. The RQNN filter shows a significant result for noise to signal ration for EEG signal filtering comparing to the RNN filter approach [3]. The major disadvantage of the neural networkbased filters that they are hard to be implemented on a wearable hardware chip with size implementation limits due to the huge requirement of wires and circuits to implement the neural network, so that is it not suitable for multiple medical applications such as seizure prediction chips [22, 15] and 
Alzheimer's disease detection [18]. However it can be applied at the BCI game applications where there are no restrictions on the hardware implementation size.

\subsection{Spatial filtering}

Spatial filtering concept is to use a small number of new channels that are a linear combination of the original channels of EEG brain signal reading [20]. Generally, the spatial filter is defined as the following:

$$
\tilde{x}=\sum_{i} w_{i} x_{i}=w X
$$

Where $\tilde{x}$ the spatially filtered signal, xi the EEG signal from channel $\mathrm{i}, w_{i}$ the weight given to that channel in the spatial filter and $\mathrm{X}$ is the original matrix of EEG brain signals from all channels.

Spatial filtering has a neurophysiological meaning because it helps to recover brain original signal by gathering the relevant information that is spread over different EEG channels. In addition, it reduces the dimensions of the EEG channels to less number of spatially filtered signals. So that spatial filtering can be used for space reduction, filtering the signal and recover the original brain signal simultaneously.

\subsection{Cauchy-Based Filter}

The Cauchy-based filter technique presented at [15] is used at seizure detection chip. This filter hardware implementation can be applied under the size limitation of BCI chips for brain medical detection chips. The proposed Cauchy distributionbased filter targets the impulsive observation noises since it is a heavy-tailed distribution which is more powerful than the Gaussian distribution to capture abrupt changes in noise. Cauchy distribution characterizes the impulsive artifacts in EEG observation such as EMG and EOG. The Cauchy distribution probability density function is:

$$
C(\tilde{x} ; \mu, s)=\frac{1}{s \pi\left(1+((\tilde{x}-\mu) / s)^{2}\right)}
$$

Where $\mu$ is the location parameter denoting the location of the peak of Cauchy distribution and $s$ is the scale parameter that specifies the half-width at half-maximum.

The noise observation function is defined at the following:

$$
\boldsymbol{z}_{t}=\boldsymbol{W} \boldsymbol{x}_{t}+\boldsymbol{u}_{t}, \quad \boldsymbol{u}_{t} \sim C(\tilde{x} ; \mathbf{0}, \boldsymbol{s})
$$

Where $\mathbf{z}_{\mathbf{t}}$ is a $\mathrm{n}$-dimensional feature vector standing for an observation at time $t, \boldsymbol{x}_{\boldsymbol{t}}$ is defined as:

$$
\boldsymbol{x}_{t}=\left(x_{t}^{0}, x_{t}^{1}, \ldots, x_{t}^{q}\right)^{T}
$$

Where $x_{t}^{i}$ denotes $\mathrm{x}_{\mathrm{t}}$ to the power $i, W$ is an $\mathrm{n} \times(\mathrm{q}+1)$ matrix containing coefficients of the polynomial function, and $\mathbf{u}_{\mathbf{t}}$ is the observation noise of the signal.

EOG and EMG effect with abrupt changes on the EEG brain signal such as large amplitudes and high frequencies. Cauchy filter able efficiently to filter these artifacts especially at medical applications such as Epilepsy Seizures.

\section{CONCLUSION}

EEG brain signal filtering is a significant step at BCI preprocessing stage to prepare an efficient signal for detection and classification learning stages. In order to effectively choose and use methods for dealing with artifact removal of EEG brain signal, the BCI application should be decided and all the hardware constraints should be determined to manipulate to select the convenient technique for EEG brain signal filtering and preprocessing. The BCI applications have specific needs for several artifact removals from EEG brain signal recording. This paper show the most remarkable filter approaches for different BCI applications. The medical applications especially that are related to on-line disease detection such as epilepsy seizure have limited restrictions on the hardware implementation. In such case a good filtering technique with light-weight hardware implementation is needed. The game application and security applications that have no limits on the hardware implementation can use more powerful filtering techniques such as neural networks.

\section{REFERENCES}

[1] E. A. Curran and S. J. Maria, S. J, "Learning to control brain activity: A review of the production and control of EEG components for driving brain-computer interface (BCI) systems", Brain and Cognition, vol. 51, no. 3, April 2003, pp. 326-336.

[2] M. Honing and D. Messerschmitt, "Adaptive Filters: Structures, Algorithms and Applications", ISBN-13: 9780898381634, 1984, Kluwer Academic Publishers.

[3] V. Gandhi, V. Arora, G. Prasad, D. Coyle and T. M. McGinnity, "A novel EEG signal enhancement approach using a recurrent quantum neural network for a braincomputer interface," in Proc. 3rd Eur. Conf., Tech. Assist. Rehabil., Mar. 2011, pp. 1-8.

[4] Z. S. Zaghloul and M. Bayoumi, "Implementable Spike Sorting techniques for VLSI wireless BCI/BMI implants: A survey," 5th International Conference on Energy Aware Computing Systems \& Applications, Cairo, 2015, pp. 1-4

[5] P. Horki, T. Solis-Escalante, C. Neuper, "Combined motor imagery and SSVEP based BCI control of a $2 \mathrm{DoF}$ artificial upper limb", Medical \& Biological Engineering \& Computing, May 2011, vol. 49, no. 5, pp 567-577

[6] A. Jackson, C. T. Moritz, J. Mavoori, T. H. Lucas and E. E. Fetz, "The neurochip BCI: towards a neural prosthesis for upper limb function," in IEEE Transactions on Neural Systems and Rehabilitation Engineering, vol. 14, no. 2, June 2006, pp. 187-190.

[7] Z. S. Zaghloul and M. Bayoumi, "Toward fast low power adaptive spike sorting VLSI chip design for wireless BCI implants," 2015 IEEE 58th International Midwest Symposium on Circuits and Systems (MWSCAS), Fort Collins, CO, 2015, pp. 1-4.

[8] Y. Chae, J. Jeong and S. Jo, "Toward Brain-Actuated Humanoid Robots: Asynchronous Direct Control Using an EEG-Based BCI," in IEEE Transactions on Robotics, vol. 28, no. 5, Oct. 2012, pp. 1131-1144.

[9] C. Guger, S. Daban, , E.Sellers, C. Holzner, G. Krausz, R. Carabalona, F. Gramatica, G. Edlinger, "How many people are able to control a P300-based brain-computer interface (BCI)?, " Neuroscience Letters, vol. 462, no.1, 18 September 2009, pp. 94-98.

[10] S. Abdulkader, A. Atia , M. M.Mostafa, "Brain computer interfacing: Applications and challenges," Egyptian Informatics Journal, vol. 16, no. 2, July 2015, pp. 213230 . 
[11] Z. Saad Zaghloul and M. Bayoumi, "Adaptive neural matching online spike sorting VLSI chip design for wireless BCI implants," 2015 IEEE International Conference on Acoustics, Speech and Signal Processing (ICASSP), South Brisbane, QLD, 2015, pp. 977-981.

[12] D. Nurseitov, A. Serekov, A. Shintemirov and B. Abibullaev, "Design and evaluation of a P300-ERP based BCI system for real-time control of a mobile robot," 2017 5th International Winter Conference on BrainComputer Interface (BCI), Sabuk, 2017, pp. 115-120.

[13] M. van de Velde, G. van Erp, PJ. Cluitmans PJ., "Detection of muscle artifact in the normal human awake EEG," Electroencephalography and Clinical Neurophysiology, vol. 107, no. 2, April 1998, pp. 149158.

[14] M. K. Bashar, I. Chiaki and H. Yoshida, "Human identification from brain EEG signals using advanced machine learning method EEG-based biometrics," 2016 IEEE EMBS Conference on Biomedical Engineering and Sciences (IECBES), Kuala Lumpur, 2016, pp. 475-479.

[15] Y. Wang et al., "A Cauchy-Based State-Space Model for Seizure Detection in EEG Monitoring Systems," in IEEE Intelligent Systems, vol. 30, no. 1, pp. 6-12, Jan.-Feb. 2015.

[16] D. Coyle, J. Principe, F. Lotte and A. Nijholt, "Guest Editorial: Brain/neuronal - Computer game interfaces and interaction," in IEEE Transactions on Computational Intelligence and AI in Games, vol. 5, no. 2, pp. 77-81, June 2013.

[17] L. Liao, C. Chen, I. Wang, S. Chen, S. Li, B. Chen, J. Chang and C. Lin, "Gaming control using a wearable and wireless EEG-based brain-computer interface device with novel dry foam-based sensors," Journal of NeuroEngineering and Rehabilitation, vol. 9, no. 5, January 2012, pp. 1-11.

[18] W. L. Woon, A. Cichocki, F. Vialatte and T, Musha, "Techniques for early detection of Alzheimer's disease using spontaneous EEG recordings, "Physiological Measurement, vol. 28, 2007, pp. 335-347.

[19] R. Oostenveld, P. Fries, E. Maris, and J. Schoffelen, "FieldTrip: open source software for advanced analysis of MEG, EEG, and invasive electrophysiological data," Neuroscience, article 1, January 2011, 9 pages.

[20] J. R. Wolpawa,b, N. Birbaumerc, D. J. McFarlanda, G. Pfurtschellere, T. M. Vaughan, "Brain-computer interfaces for communication and control," Clinical Neurophysiology, vol. 113, March 2002, pp. 767-791.

[21] T. W. Picton, P. van Roon, M. L. Armilio, "The correction of ocular artifacts: a topographic perspective," Clinical Neurophysiology, vol. 111, no. 1, January 2000,pp. 53-65.

[22] N. Elsayed, Z. S. Zaghloul, M. Bayoumi, "BCI/AIS Low Power Adaptive Architecture for Early Prediction of Epilepsy Seizures," 15th IEEE International New Circuits and Systems Conference (NEWCAS), Strasbourg, France, June 2017, in press.

[23] A. GuruvaReddy, S. Narava, "Artifact Removal from EEG Signals," International Journal of Computer Applications, vol. 77, no. 13, September 2013, pp. 17-19.

[24] D. Craven, B. McGinley, L. Kilmartin, M. Glavin and E. Jones, "Adaptive Dictionary Reconstruction for Compressed Sensing of ECG Signals," in IEEE Journal of Biomedical and Health Informatics, vol. 21, no. 3, May 2017, pp. 645-654.

[25] S. R. Benbadis and K. Lin, "Errors in EEG Interpretation and Misdiagnosis of Epilepsy," European Neurology, vol. 59, 2008, pp. 267-271.

[26] D. V. Morettia, F. Babilonia, F. Carduccia, F. Cincottia, E. Remondinia, P.M. Rossinib, S. Salinarie, C. Babiloni, "Computerized processing of EEG-EOG-EMG artifacts for multi-centric studies in EEG oscillations and eventrelated potentials," International Journal of Psychophysiology, vol. 47, no. 3, March 2003, pp. 199216. 